\title{
A comparison of single factor Markov-functional and multi factor market models
}

\author{
Raoul Pietersz • Antoon Pelsser
}

Published online: 12 January 2010

(C) The Author(s) 2010. This article is published with open access at Springerlink.com

\begin{abstract}
We compare single factor Markov-functional and multi factor market models and the impact of their correlation structures on the hedging performance of Bermudan swaptions. We show that hedging performance of both models is comparable, thereby supporting the claim that Bermudan swaptions can be adequately risk-managed with single factor models. Moreover, we show that the impact of smile can be much larger than the impact of correlation. We use the constant exercise method for calculating risk sensitivities of callable products in market models, which is a modification of the least-squares Monte Carlo method. The hedge results show the constant exercise method enables proper functioning of market models as risk-management tools.
\end{abstract}

Keywords Markov-functional model · Market model - Bermudan swaption · Terminal correlation $\cdot$ Hedging $\cdot$ Greeks for callable products $\cdot$ Smile

JEL Classification G13

We are grateful to ABN AMRO Bank for supplying market data. We are also grateful for the comments of Nevena Šelić and seminar participants at the RODEO Research Forum, Antwerp, Belgium. We thank the two anonymous referees for excellent suggestions.

R. Pietersz $(\varangle)$

Product Development Group, Quantitative Analytics, Global Markets, ABN AMRO Bank, P.O. Box 283, 1000 EA Amsterdam, The Netherlands

e-mail: raoul@pietersz.org

A. Pelsser

Departments of Finance and Quantitative Economics, Maastricht University, P.O. Box 616,6200 MD Maastricht, The Netherlands

e-mail: a.pelsser@maastrichtuniversity.nl 


\section{Introduction}

Bermudan swaptions form a popular class of interest rate derivatives. The underlying is a plain-vanilla interest rate swap, in which periodic fixed payments are exchanged for floating LIBOR payments. Institutional debt issuers use interest rate swaps to revert from floating to fixed interest rate payments, and vice versa. Often the issuers want to reserve the right to cancel the swap. A cancellable swap can be valued by the following parity relation. A cancellable interest rate swap is equal to a plain-vanilla interest rate swap plus a callable interest rate swap with reversed cash flows. Thus a cancellable swap can be valued when the callable swap can be valued. Such callable swap options are referred to as Bermudan swaptions. Bermudan means that the exercise opportunities are at a discrete set of time points. A European swaption is an option to enter into a swap at only a single exercise date.

In this paper, we study the pricing and hedging performance of two popular models for Bermudan swaptions, including the impact of correlation on hedging. Many models have been proposed in the literature for valuation and risk management of Bermudan swaptions. We distinguish three categories: short-rate models, Markovfunctional models and market models.

Short-rate models model the dynamics of the term structure of interest rates by specifying the dynamics of a single rate (the short rate) from which the whole term structure at any point in time can be calculated. Examples of short-rate models include the models of Vasicek (1977), Cox et al. (1985), Dothan (1978), Black et al. (1990), Ho and Lee (1986) and Hull and White (1990).

The Markov-functional model of Hunt et al. (2000) assumes that the discount factors are a function of some underlying Markov process. The model is then fully determined by no-arbitrage arguments and by requiring a fit to the initial yield curve and interest rate option volatility.

Market models were introduced by Brace et al. (1997), Miltersen et al. (1997) and Jamshidian (1997). The name 'market model' refers to the modelling of market observable variables such as LIBOR rates and swap rates. The explicit modelling of market rates allows for natural formulas for interest rate option volatility, that are consistent with the market practice of using the formula of Black (1976) for caps (options on LIBOR) and swaptions (options on swap rates).

Short-rate and Markov-functional models are usually ${ }^{1}$ implemented as models with a single stochastic process driving the term structure of interest rates. A disadvantage is then that the instantaneous correlation between interest rates can only be 1 . Market models, however, efficiently allow for any number of stochastic variables to be used, so that any instantaneous correlation structure can be captured. There is substantial evidence that the term structure of interest rates is driven by multiple factors (three, four, or even more), see the review article of Dai and Singleton (2003). A more realistic description of reality may thus be expected from decorrelation in multi factor models, which points to possibly better hedge performance. The question addressed in this paper is whether the increase in hedge performance due to use of a multi factor

\footnotetext{
1 Two factor short rate models exist too, see for example Ritchken and Sankarasubramanian (1995).
} 
model is significant. To those that a priori dismiss the use of single factor models due to their economic irrelevance by failure in capturing the multi factor dynamics of the term structure of interest rates, we say: Models that are best for managing an interest rate derivatives book are not necessarily models that are most realistic, rather they are models that most reduce variance of profit and loss $(\mathrm{P} \& \mathrm{~L})$, thereby preserving wealth in the most stable manner. We mention five articles that compare single and multi factor models.

First, in favour of multi factor models, Longstaff et al. (2001) claim that short-rate models, because of supposedly misspecified dynamics, lead to suboptimal exercise strategies. This claim is supported by empirical evidence performed with the shortrate models of Black et al. (1990) and Black and Karasinski (1991). The authors then conclude that the costs to Wall Street firms of following single factor exercise strategies could be several billion dollars.

Second, in favour of single factor models, Andersen and Andreasen (2001) claim that the exercise strategy obtained from a properly calibrated single factor model only leads to insignificant losses when applied in a two factor model.

Third, Driessen et al. (2003) are the first to investigate hedge performance. These authors investigate two types of delta hedge instruments, (1) a number of delta hedge securities, i.e. discount bonds, equal to the number of factors, and (2) a large set of discount bonds, one for each security spanning the yield curve. They show that if the number of hedge instruments is equal to the number of factors, then multi factor models outperform single factor models. If, however, the large set of hedging instruments is used, which is the case in practice, then single factor models perform as well as multi factor models in terms of delta hedging of European swaptions.

Fourth, Fan et al. (2003) show, for the case of the number of hedge instruments equal to the number of factors, that higher factor models perform better than lower factor models in terms of delta hedging of European swaptions and European swaption straddles. ${ }^{2}$ The results of Fan et al. (2003) are thus consistent with the findings of Driessen et al. (2003).

Fifth, Gupta and Subrahmanyam (2005) show that single-factor models provide accurate pricing when calibrated to the volatility smile. For hedging, however, a second stochastic factor yields better performance than a single-factor model calibrated to smile. The primary difference relative to our paper is that Gupta and Subrahmanyam (2005) use factor hedging ("within model hedging") whereas we hedge with all instruments with which the interest curve and volatility surface are built ("outside of model hedging").

Relative to Driessen et al. (2003) and Fan et al. (2003), we make the contribution of also considering vega hedging and Bermudan-style swaptions rather than only delta hedging and only European-style swaptions. A European product depends solely on the marginal distributions of the swap rates, whereas a Bermudan product depends on correlation and the joint distribution, too. The failure of single-factor models to fully capture the correlation structure may thus potentially adversely affect hedging results, and this issue has not yet been addressed in the literature, since Driessen et al. (2003)

2 A European swaption straddle consists of a position of long a payer swaption and long an otherwise identical receiver swaption. 
and Fan et al. (2003) study either correlation-insensitive products or products that are sensitive to correlation within the model only because the model is not fully calibrated to the volatility of the canonical rate underlying the product. In contrast, we fit the models exactly to a subset of European swaptions particular to a Bermudan swaption rather than attempting a partial fit to the whole swaption volatility surface, as Driessen et al. (2003) and Fan et al. (2003). The two practices of (1) fitting to an appropriate set of swaptions, and (2) vega hedging, are probably more close in spirit to financial practice. In fact, we show that the variance of $P \& L$ is significantly reduced when a vega hedge has been set up additional to a delta hedge.

There is one drawback of using high factor models, however, which is lesser tractability than low (one or two) factor models. For valuation in high factor models, we must resort to Monte Carlo (MC) simulation. Valuation by MC is not a problem, but the estimation of sensitivities (Greeks) can be less efficient. This is not due to the choice of calibration, as can sometimes be the case as shown by Pietersz and Pelsser (2004), since in this paper the safe option of time-constant volatility (but dependent on the forward rates) is used. The less efficient estimation of sensitivities occurs if the payoff along the path can change discontinuously as dependent on initial parameters, see, for example, Glasserman (2004, Section 7.1). We show that such discontinuity appears in the Longstaff and Schwartz (2001) algorithm for valuation of Bermudan-style options. We consider two methods to improve the efficiency of sensitivity estimates. The comparison of hedge performance of single and multi factor models thus entails a trade-off between more realistic correlation modelling and tractability.

For the Markov-functional model, the failure of not capturing a realistic instantaneous correlation structure can be remedied, in some sense, for Bermudan swaptions and perhaps for other derivatives, too, as follows. In theory the price of a co-terminal Bermudan swaption is dependent of and fully determined by the joint distribution of the forward co-terminal swap rates at each of the exercise dates. In effect there are thus $n(n+1) / 2$ stochastic variables that determine the price. In this paper, we use the observation that the price of a Bermudan swaption is, up to first order approximation, determined by the joint distribution of only the underlying spot co-terminal swap rates at the exercise dates, see, e.g., Piterbarg (2004b, p. 67). There are only $n$ such spot co-terminal swap rates. The marginal distributions of these swap rates are governed by the associated European swaption volatility quoted in the market, whereby, in a log-normal model, we only need to specify correlation. We will call their correlation the terminal correlation. A novel approximating formula is derived for the terminal correlation in the Markov-functional model. The accuracy of the new formula is tested numerically. The novel formula allows the Markov-functional model to be calibrated to terminal correlation. We then equip a full factor swap market model with a parameterized instantaneous correlation matrix, calculate the resulting terminal correlation and fit the Markov-functional model to this terminal correlation. Thus, although the Markov-functional model fails to capture instantaneous correlation, it can be tweaked such that it is fitted to product specific terminal correlation. Since such correct correlation specification more or less determines the price of the Bermudan swaption, it then no longer matters for pricing Bermudan swaptions whether the single factor Markov-functional model is a realistic or unrealistic model of other parts of reality in the interest rate market, outside of the volatilities and correlations of the relevant 
swap rates. Essentially, we have projected all relevant parts of reality correctly onto the single factor Markov-functional model. With the thus fitted Markov-functional model, and also with swap and LIBOR market models, we subsequently compare hedge performance of Bermudan swaptions with real market data over a 1 year period.

By fitting to terminal correlation in Markov-functional models we loose the freedom to specify time dependency of volatility. In contrast, market models allow specification of time dependent correlation without affecting time dependency of volatility. We also study the impact of time dependent correlation on hedging performance in market models.

The research in this paper is not aimed at comparing the model generated Bermudan swaption prices to real-life market quoted prices. Rather, the hypothetical viewpoint is taken that swaps and European swaptions are liquidly traded in the market, and Bermudan swaptions are less liquidly traded. The model is then used as an extrapolation tool to determine a Bermudan swaption price consistent with swap and European swaption prices, and such that the risk sensitivities provide a hedge of the former in terms of the latter securities. In any case, the study in this paper is relevant for non-standard Bermudan swaptions, for which the underlying has more exotic coupon payments. Examples of such exotic coupon payments are capped floater $(\min (\ell L, K)$ for some cap rate $K$ and leverage $\ell$ ), inverse floater $(\max (K-\ell L, 0))$ and range accrual ( $\alpha L$, with $\alpha$ the fraction for which LIBOR within the accrual period is within a certain range). These non-standard Bermudan swaptions are called callable LIBOR exotics. The results of this paper may apply to many types of callable LIBOR exotics, but further research will have to provide a definitive answer. Nonetheless, the results of this paper are interesting for the study of callable LIBOR exotics, since these have evolved from standard Bermudan swaptions.

It is very much relevant to compare hedge performance across models and correlation specifications. Market data may lack for Bermudan swaptions. Perhaps some institutions have access to Bermudan swaption market data, but we may safely state that market data for callable LIBOR exotics most often lacks in current market environments. When market data lacks, then models are employed for valuation and hedging purposes. A model then truly governs the profit and loss of the financial institution with respect to the Bermudan swaption; we are dealing with genuine book values. A different choice of model or of correlation specification may thus lead to different behaviour of book value variability.

For both the swap market model and the Markov-functional model we initially use the basic well-known non-smile versions. Smile is the phenomenon that for European options different Black-implied volatility is quoted for different strikes of the option. As mentioned in Hunt et al. (2000, last paragraph of Section 3.2), the Markovfunctional model can be fitted to smile. We provide details, also for the swap market model, and show that the resulting smile-fitting procedure is numerically efficient and straightforward to implement. The smile Markov-functional model and smile swap market model are subsequently fitted to USD swaption smile data. We then compare empirically the impact of smile versus the impact of correlation.

The LIBOR Markov-functional model has been compared with the LIBOR market model before by Bennett and Kennedy (2005). These authors show that the one factor LIBOR Markov-functional model with mean reversion and the one factor separable 
LIBOR market model are largely similar in terms of dynamics and pricing. They also show this for an approximated version of the LIBOR market model by drift approximations, as introduced by Pietersz et al. (2004) and Hunter et al. (2001). Relative to Bennett and Kennedy (2005) this paper makes the contribution of also comparing multi factor models with the Markov-functional model. Moreover, we show how multi factor models can a priori be compared to the Markov-functional model which is not a straightforward extension from the one-dimensional case.

The study in this paper relates to the debate about suboptimality of single-factor exercise strategies for Bermudan swaptions, where Longstaff et al. (2001) claim single-factor exercise strategies lead to significant misvaluations whereas Andersen and Andreasen (2001) and Svenstrup (2005) claim only insignificant misvaluations occur. We show that the difference between single-factor and multi-factor exercise strategies is insignificant for Bermudan swaptions not only for valuation performance but also for hedging performance.

The remainder of the paper is organized as follows. First, we outline the comparison methodology for the two models. The LIBOR and swap market models and Markovfunctional model are discussed, as well as the two Greeks calculation methods for market models. Second, the data is described. Third, we numerically test the accuracy of an approximating formula for the terminal correlation in the Markov-functional model. Fourth, empirical comparison results are presented. Fifth, the impact of smile is investigated. Sixth, we conclude.

\section{Methodology}

In this section, we first introduce some notation. Second, we set up the framework that enables a comparison between multi factor and single factor models.

The type of Bermudan swaption that is considered here is the co-terminal version, as opposed to, for example, the fixed maturity version. A co-terminal Bermudan swaption is an option to enter into an underlying swap at several exercise opportunities, where each swap ends at the same contractually determined end date. The maturity of the swap entered into thus becomes smaller as the option is exercised later. In contrast, for a fixed maturity Bermudan swaption, each swap that can be entered into has the same contractually specified maturity and the respective end dates then differ. We consider a Bermudan swaption on an underlying swap with $n$ payments and a fixed rate $F$. Associated with this swap is a tenor structure $0<t_{1}<\cdots<t_{n+1}$. The underlying swap makes a payment $P_{i}$ at time $t_{i+1}$ depending on the LIBOR rate $L\left(t_{i}\right)$ fixed at time $t_{i}$ for $i=1, \ldots, n$. Denote the notional amount by $N$ and the day count fraction for accrual period $\left[t_{i}, t_{i+1}\right]$ by $\alpha_{i}$. Introduce the variable $\phi \in\{-1,1\}$ by $\phi=1$ for a pay fixed swap and $\phi=-1$ for a receive fixed swap. The payment $P_{i}$ is then $\phi \alpha_{i}\left(L\left(t_{i}\right)-F\right) N$. The holder of the Bermudan swaption has the right to enter into the swap at the dates $t_{1}, \ldots, t_{n}$. If the holder exercises the option at time $t_{i}$, then he or she will receive the payments $P_{i}, \ldots, P_{n}$. Alternatively, in the market the holder could have entered into an otherwise equal swap but with fixed rate equal to the swap rate $S_{i: n}\left(t_{i}\right)$. Here $S_{i: j}$ denotes the forward swap rate for a swap that start at $t_{i}$ and ends at $t_{j+1}$. The holder will thus only exercise the Bermudan at time $t_{i}$ if $\phi\left(S_{i: n}\left(t_{i}\right)-F\right)>0$. But even when 
the immediate exercise value is positive, the holder can nonetheless decide to hold on to the option in view of a more favourable forward swap rate $S_{j: n}\left(t_{i}\right), j>i$. It follows that the price of a Bermudan swaption is dependent of and fully determined by the joint distribution of the variables $\left\{S_{j: n}\left(t_{i}\right) ; j=i, \ldots, n, i=1, \ldots, n\right\}$. The forward swap rates $\left\{S_{1: n}, \ldots, S_{n: n}\right\}$ are called co-terminal since they all co-end at the same termination date.

We contend that the main driver for the price of Bermudan swaptions is the joint distribution of the realizations of the co-terminal swap rates $\left\{S_{i: n}\left(t_{i}\right) ; i=1, \ldots, n\right\}$. Ostrovsky (2002) calls this the diagonal process. The economic argument is that prima facta, the holder of the option has to choose between receiving the payoffs of entering into the swaps starting at $t_{1}, t_{2}, \ldots, t_{n}$ and the associated payoffs are determined fully by $S_{1: n}\left(t_{1}\right), S_{2: n}\left(t_{2}\right), \ldots, S_{n: n}\left(t_{n}\right)$.

As is common in financial practice, we calibrate models to only those sections of the market that are relevant to the product, rather than attempting to fit the models to all available market data. We assume that any valuation model for the Bermudan swaption is calibrated to the so-called diagonal of European swaptions that start at $t_{i}$ and end at $t_{n+1}, i=1, \ldots, n$. This means that the variance of the variables $\left\{S_{1: n}\left(t_{1}\right), \ldots, S_{n: n}\left(t_{n}\right)\right\}$ is already fully determined. Thus the diagonal process is fully determined (given a normal or log-normal distribution) if we specify the correlation matrix for the variables $\left\{S_{i: n}\left(t_{i}\right) ; i=1, \ldots, n\right\}$. This correlation matrix will be called the terminal correlation. In the next three sections, we discuss the LIBOR and swap market models and the Markov-functional model, respectively. We show how the terminal correlation can approximately be calculated in the swap market model and the Markov-functional model. For the Markov-functional model we show how the model can be calibrated to the terminal correlation.

The idea of terminal correlation is not new to finance. For example, Rebonato (2002, Section 7.1.2) shows that it is the terminal and not the instantaneous correlation that directly affects the price of swaptions. The terminal correlation itself is determined both by the instantaneous correlation and the term structure of instantaneous volatility. In Rebonato (1999, Section 11.4) it is shown that the terminal correlation is influenced just as much, and even more, by the instantaneous volatility than by the instantaneous correlation.

\subsection{The LIBOR and swap market models}

Within the swap market model, $n$ forward swap rates are modelled as log-normal processes under their respective forward measure, with forward swap rate $S_{i: n}$ satisfying,

$$
\frac{\mathrm{d} S_{i: n}(t)}{S_{i: n}(t)}=\sigma_{i: n}(t) \mathrm{d} W^{(i: n)}(t), \quad\left\langle\mathrm{d} W^{(i: n)}(t), \mathrm{d} W^{(j: n)}(t)\right\rangle=\rho_{i: n, j: n}(t) \mathrm{d} t .
$$

Here $\sigma_{i: n}(\cdot)$ denotes the instantaneous volatility function and $W^{(i: n)}$ denotes a Brownian motion under the $i^{\text {th }}$ forward swap measure. The latter measure is associated with a portfolio of discount bonds, weighted by the respective day count fractions, with 
maturity times corresponding to the payment times of the swap. The value of such a portfolio of discount bonds is named the present value of a basis point (PVBP).

Within the LIBOR market model, $n$ forward LIBORs are modelled as log-normal processes under their respective forward measure, with forward LIBOR $L_{i}$ satisfying,

$$
\frac{\mathrm{d} L_{i}(t)}{L_{i}(t)}=\sigma_{i}(t) \mathrm{d} W^{(i+1)}(t), \quad\left\langle\mathrm{d} W^{(i+1)}(t), \mathrm{d} W^{(j+1)}(t)\right\rangle=\rho_{i j}(t) \mathrm{d} t .
$$

Here $\sigma_{i}(\cdot)$ denotes the instantaneous volatility function and $W^{(i+1)}$ denotes a Brownian motion under the $i^{\text {th }}$ forward measure. The latter measure is associated with a discount bond that matures at $t_{i+1}$, the payment time of the $i^{\text {th }}$ LIBOR deposit. The LIBOR market model is calibrated approximately to swaption volatility, via an approximation of swaption volatility in terms of LIBOR volatility, see, e.g., Hull and White (2000). By assumption of constant volatility and constant correlation (see below), the resulting calibration algorithm reduces to a simple bootstrap algorithm for determining the LIBOR volatility levels.

Within both market models, we set the instantaneous volatility and correlation constant over time, i.e., $\sigma_{i: n}(t)=\sigma_{i: n}$ and $\rho_{i: n, j: n}(t)=\rho_{i: n, j: n}$ for the swap model, and $\sigma_{i}(t)=\sigma_{i}$ and $\rho_{i j}(t)=\rho_{i j}$ for the LIBOR model. These choices, relative to the timehomogeneous case, will not, or only favourably, impact the results, as explained by the following two arguments. First, a constant instantaneous volatility assumption leads to efficiently estimated risk sensitivities, whereas certain specific time-homogeneous specifications may not, as shown by Pietersz and Pelsser (2004). Second, our choice of parametrization of the correlation matrix is both a constant and time-homogeneous parametrization. Moreover, in Sect. 5.6 below we assess the impact of time varying correlation.

The rank of the correlation matrix $R=\left(\rho_{i j}\right)_{i, j=1}^{n}$ determines the number of Brownian motions (number of factors) driving the model. When an arbitrary correlation matrix has been specified, generally such matrix has full rank $n$, but then if a number of factors $k<n$ be required, we are led to solve a rank reduction problem. ${ }^{3}$ To test the two extreme cases, we consider only either rank 1 or full-rank correlation matrices, allowing, respectively, correlation constant at 1 or a full fit to any correlation matrix.

We parameterize the instantaneous correlation matrix by, for $i<j$,

$$
\rho_{i j}(a)=\sqrt{\frac{\left(e^{2 a t_{i}}-1\right) / t_{i}}{\left(e^{2 a t_{j}}-1\right) / t_{j}}} \text { for } a>0, \text { and } \rho_{i j}(a) \equiv 1, \text { for } a=0 .
$$

This parametrization of instantaneous correlation allows for a simple calibration of the Markov-functional model to the terminal correlation of the swap market model. In fact, parametrization (1) has been chosen such that the resulting terminal correlation of the swap market model exactly matches the terminal correlation of a Markovfunctional model with mean reversion parameter $a$. The correlation structure (1) is

\footnotetext{
3 For solving such rank reduction problems the reader is referred to Pietersz and Groenen (2004a), Pietersz and Groenen (2004b), Grubišić and Pietersz (2007), Wu (2003), Rebonato (2002, Section 9) or Brigo (2002).
} 


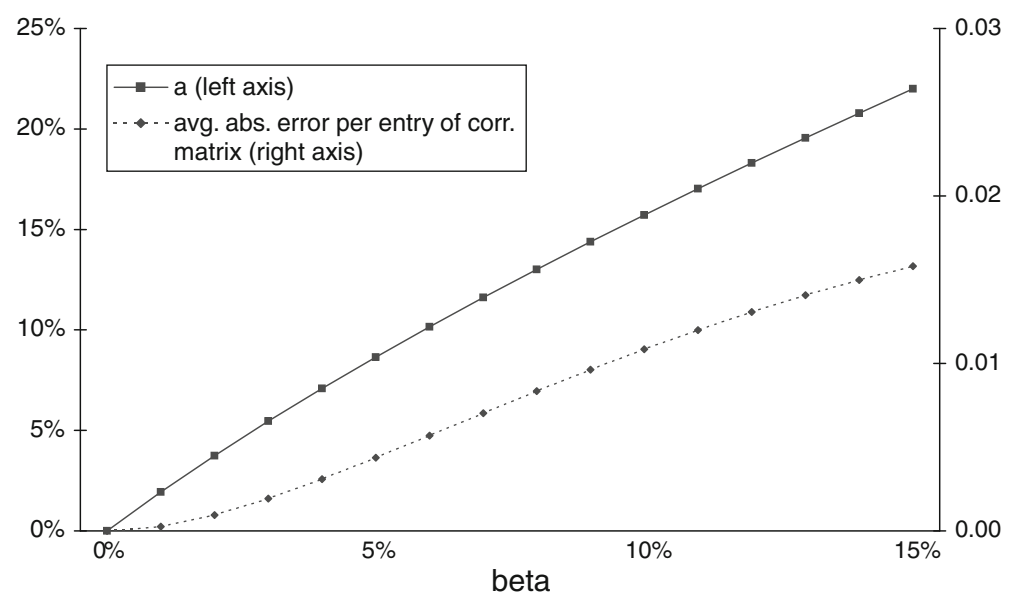

Fig. 1 Fitted $a$-parameter of parametrization $\rho_{i j}(a)=\sqrt{ }\left(\left(\left(\exp \left(2 a t_{i}\right)-1\right) / t_{i}\right) /\left(\left(\exp \left(2 a t_{j}\right)-1\right) / t_{j}\right)\right)$ (left axis) and fit error (right axis) versus the $\beta$-parameter of the Rebonato (1998) parametrization $\rho_{i j}(\beta)=\exp \left(-\beta\left|t_{i}-t_{j}\right|\right)$ The fit error is the average absolute error over the entries

nonetheless a good choice, since we will show that, for a suitable choice of $a$, (1) corresponds to a form that is often quoted in the literature, see, for example, Rebonato (1998, Eq. (4.5), p. 83),

$$
\rho_{i j}(\beta)=\exp \left(-\beta\left|t_{i}-t_{j}\right|\right), \quad \text { for some } \beta \geq 0 .
$$

We numerically fitted the form of (1) to (2), for $10 \times 10$ correlation matrices, where $n=10$ corresponds to the setting in the forthcoming hedge tests. In other words, fix $\beta$, and then find $a$ that solves

$$
\min _{a \geq 0} \sum_{i=1}^{n} \sum_{j=1}^{n}\left|\rho_{i j}(a)-\rho_{i j}(\beta)\right| .
$$

The relationship between the fitted $a$ as dependent on $\beta$ has been displayed in Fig. 1 . As can be seen from the figure, the fit is of good quality, obtaining an average absolute error over the entries in the correlation matrix that is less than 0.02 for typical values of $\beta$ and $a$.

\subsection{The Markov-functional model}

We consider the swap variant of the Markov-functional model, see Hunt et al. (2000, Section 3.4) for details on this variant. Within the (swap) Markov-functional model, any model variable is a function of an underlying Markov process $x$. For example, for a forward swap rate we have $S_{i: n}\left(t_{j}\right)=S_{i: n}\left(t_{j}, x\left(t_{j}\right)\right)$. We assume that the driving Markov process of the model is a deterministically time-changed Brownian motion, satisfying 


$$
\mathrm{d} x(t)=\tau(t) \mathrm{d} W(t)
$$

Here $\tau(\cdot)$ denotes a deterministic function (that can be chosen piece-wise constant) and $W$ denotes a Brownian motion.

We now present an approximate formula for the terminal correlation. An argument explaining the formula is given, and in a later section we investigate the accuracy of the approximating formula. By a Taylor expansion, we have $\ln S_{i: n}\left(t_{i}, x\right) \approx$ $s_{i: n}^{(0)}\left(t_{i}\right)+s_{i: n}^{(1)}\left(t_{i}\right) x$. Since correlation is unaltered by a linear transformation, the terminal correlation of the swap rates is thus approximately equal to the terminal correlation of the underlying Markov process,

$$
\rho\left(\ln S_{i: n}\left(t_{i}\right), \ln S_{j: n}\left(t_{j}\right)\right) \approx \rho\left(x\left(t_{i}\right), x\left(t_{j}\right)\right) .
$$

By straightforward calculation, for $i<j$,

$$
\rho\left(x\left(t_{i}\right), x\left(t_{j}\right)\right)=\frac{\operatorname{Cov}\left(x\left(t_{i}\right), x\left(t_{j}\right)\right)}{\sqrt{\operatorname{Var}\left(x\left(t_{i}\right)\right) \operatorname{Var}\left(x\left(t_{j}\right)\right)}}=\sqrt{\frac{\int_{0}^{t_{i}} \tau^{2}(t) \mathrm{d} t}{\int_{0}^{t_{j}} \tau^{2}(t) \mathrm{d} t}} .
$$

In fact, any functional of the Markov process can be linearized by a Taylor expansion and, according to the argument above, would exhibit the same approximate terminal correlation (4). The above theoretical argument is therefore not very strong. The approximation, however, turns out to be accurate, as will be shown numerically in Sect. 4.

In principle, the Markov-functional model can thus be approximately fitted to the terminal correlation by minimization of the fitting error given a market-implied or historically estimated terminal correlation matrix. The parameters for this minimization problem are for example the $n$ parameters governing the piece-wise constant function $\tau(\cdot)$. For ease of exposition we will, however, restrict our attention to the case of mean reversion, i.e. $\tau(t)=\exp (a t)$, with $a$ denoting the mean reversion parameter, see Sect. 4 of Hunt et al. (2000). In this case we have, for $i<j$,

$$
\rho\left(x\left(t_{i}\right), x\left(t_{j}\right)\right)=\sqrt{\frac{e^{2 a t_{i}}-1}{e^{2 a t_{j}}-1}} .
$$

To verify that the Markov-functional model is properly calibrated to terminal correlation, in the swap market model this correlation is approximately calculated to be, from (1), for $i<j$,

$$
\frac{\int_{0}^{t_{i}} \sigma_{i: n}(t) \sigma_{j: n}(t) \rho_{i j}(t) \mathrm{d} t}{\sqrt{\int_{0}^{t_{i}} \sigma_{i: n}^{2}(t) \mathrm{d} t \int_{0}^{t_{j}} \sigma_{j: n}^{2}(t) \mathrm{d} t}}=\frac{\sigma_{i: n} \sigma_{j: n} \rho_{i j} t_{i}}{\sqrt{\sigma_{i: n}^{2} t_{i} \sigma_{j: n}^{2} t_{j}}}=\rho_{i j} \sqrt{\frac{t_{i}}{t_{j}}}=\sqrt{\frac{e^{2 a t_{i}}-1}{e^{2 a t_{j}}-1}} .
$$

The specification (1) of the instantaneous correlation of the swap market model was constructed such that the (approximate) terminal correlation (5) of the Markov-functional model with mean reversion parameter $a$ is equal to the (approximate) terminal 
correlation (6) in the swap market model with parameter $a$. Note that this correspondence does not necessarily hold for the LIBOR market model, though we nonetheless employ it in the comparison tests.

\subsection{Estimating Greeks for callable products in market models}

The algorithm of Longstaff and Schwartz (2001) (LS) renders the numeraire relative payoff along a simulated path discontinuously dependent on initial input. The discontinuity in the LS algorithm stems from the estimated optimal exercise index chosen from a discrete set of possible exercise opportunities. Such a discrete choice is inherently discontinuously dependent on initial input. Any discontinuity in a simulation may cause finite difference estimates of sensitivities to be less efficient, see Glasserman (2004, Section 7.1). We describe two methods that enhance the efficiency of finite difference estimates. These are:

(1) Finite differences with optimal perturbation size.

(2) Constant exercise method.

The two methods are discussed below in more detail. We denote by $V$ the base value of the derivative, i.e., the value of the derivative in the unperturbed model.

Method (1), the finite differences method is best described as the bump-and-revalue approach. Initial market data is perturbed by amount $\varepsilon$, the model is re-calibrated and subsequently priced at $V(\varepsilon)$. The finite difference estimate of the Greek is then $(V(\varepsilon)-V) / \varepsilon$. The mean square error (MSE) of the finite difference estimator is dependent on the chosen perturbation size $\varepsilon$. If the numeraire relative payoff along the path is continuously dependent on initial input, then least MSE is obtained when $\varepsilon$ is selected as small as possible (though larger than machine precision), see Glasserman (2004). If the payoff is discontinuous, however, then there is a trade-off between increasing and decreasing $\varepsilon$, leading to an optimal ('large' and positive) choice of $\varepsilon$ that attains least MSE, see Glasserman (2004). After some preliminary testing, we found perturbation sizes of roughly 1 basis point (bp, $0.01 \%$ ) for delta and $5 \mathrm{bp}$ for vega.

Method (2) we consider, is named the constant exercise method. Here, for the base valuation we record per path when the exercise decision takes place. In the perturbed model, we no longer perform LS least-squares Monte Carlo, but rather use the very same exercise strategy as in the base valuation case. The constant exercise method, introduced in Piterbarg (2003), provides stable and unbiased estimate of risk sensitivities, see Piterbarg (2004a, Proposition 2) for a formal proof. Moreover, the method is straightforward to implement, and more efficient, since in re-valuations linear regressions for the LS algorithm are no longer required. Note that the constant exercise method renders a re-valuation continuously dependent on initial market data, provided the underlying swap payoff is continuous, which is the case for the Bermudan swaption studied in this paper. From the discussion on perturbation sizes for method (1), it then follows that a least-MSE finite difference estimate of sensitivities is obtained by employing perturbation sizes that are as small as possible. We use $10^{-5}$ bp for both delta and vega.

We end this section by a brief discussion of other methods for calculation of Greeks available in the literature. These methods could not straightforwardly be extended to 
the situation of our investigations. Discussed are the path-wise method Glasserman and Zhao (1999), the likelihood ratio method Glasserman and Zhao (1999), the Malliavin calculus approach Fournié et al. (1999) and the utility minimization approach Avellaneda and Gamba (2001). The path-wise method cannot handle discontinuous payoffs. The likelihood ratio and Malliavin calculus method both require that the matrix of instantaneous volatility be invertible. For the market model setting, we have an $n \times d$ matrix with $n$ the number of forward rates and $d$ the number of stochastic factors. Usually $d<n$ and most often $d \ll n$, which rules out inverting the instantaneous volatility matrix. Glasserman and Zhao (1999, Section 4.2) have resolved the non-invertibility issue only for a particular case, that does not apply to our case: When the payoff is dependent only on the rates at their fixing times, $\left\{S_{1: n}\left(t_{1}\right), S_{2: n}\left(t_{2}\right), \ldots, S_{n: n}\left(t_{n}\right)\right\}$. Finally, the utility minimization approach simply calculates a different sort of risk sensitivity and is thus altogether biased.

\section{Data}

We describe the data used in the empirical comparison and smile-impact tests. All market data was kindly provided by ABN AMRO Bank.

First, we describe the data used in the comparison test. For the comparison test, we use an arbitrarily chosen time-span, 16 June 2003-2004, of USD data of mid-quotes for deposit rates, swap rates and at-the-money (ATM) swaption volatility. We use the 1 and 12 months deposit rates and the 2Y, 3Y, 4Y, 5Y, 7Y, 10Y and $15 \mathrm{Y}$ swap rates. The discount factors are bootstrapped from market data. Any discount factors required at dates not available from the bootstrap are calculated by means of linear interpolation on zero rates. A statistical description of the swaption volatility data is displayed in Table 1 . Any volatility required at expiries and tenors not available from Table 1 are calculated by means of linear surface interpolation.

Second, we describe the data used in the smile-impact test, in which we will consider a 6 year deal. We use USD data for 21 February 2003. The discount factors have been displayed in Table 2 . The swaption volatility against strike and expiry has been displayed in Table 3.

\section{Accuracy of the terminal correlation formula}

The terminal correlation in the Markov-functional model is estimated via the terminal covariance. We have, for $i<j$, for any measure,

$$
\mathbb{E}\left[\ln S_{i: n}\left(t_{i}\right) \ln S_{j: n}\left(t_{j}\right)\right]=\mathbb{E}\left[\ln S_{i: n}\left(t_{i}\right) \mathbb{E}\left[\ln S_{j: n}\left(t_{j}\right) \mid \mathcal{F}\left(t_{i}\right)\right]\right] .
$$

The above equality follows from the $\mathcal{F}\left(t_{i}\right)$-measurability of $\ln S_{i: n}\left(t_{i}\right)$. Expression (7) can be calculated on a lattice. We estimate (7) by calculating for each grid point at time $t_{i}$ the conditional expectation $\mathbb{E}\left[\ln S_{j: n}\left(t_{j}\right) \mid \mathcal{F}\left(t_{i}\right)\right]$, subsequently we integrate the result multiplied by $\ln S_{i: n}\left(t_{i}\right)$ to obtain the required expectation.

The accuracy of the approximate formula (3) is tested for a 40 years deal, with EUR market data of 8 February 1999, for which the swaption volatility level is on average 


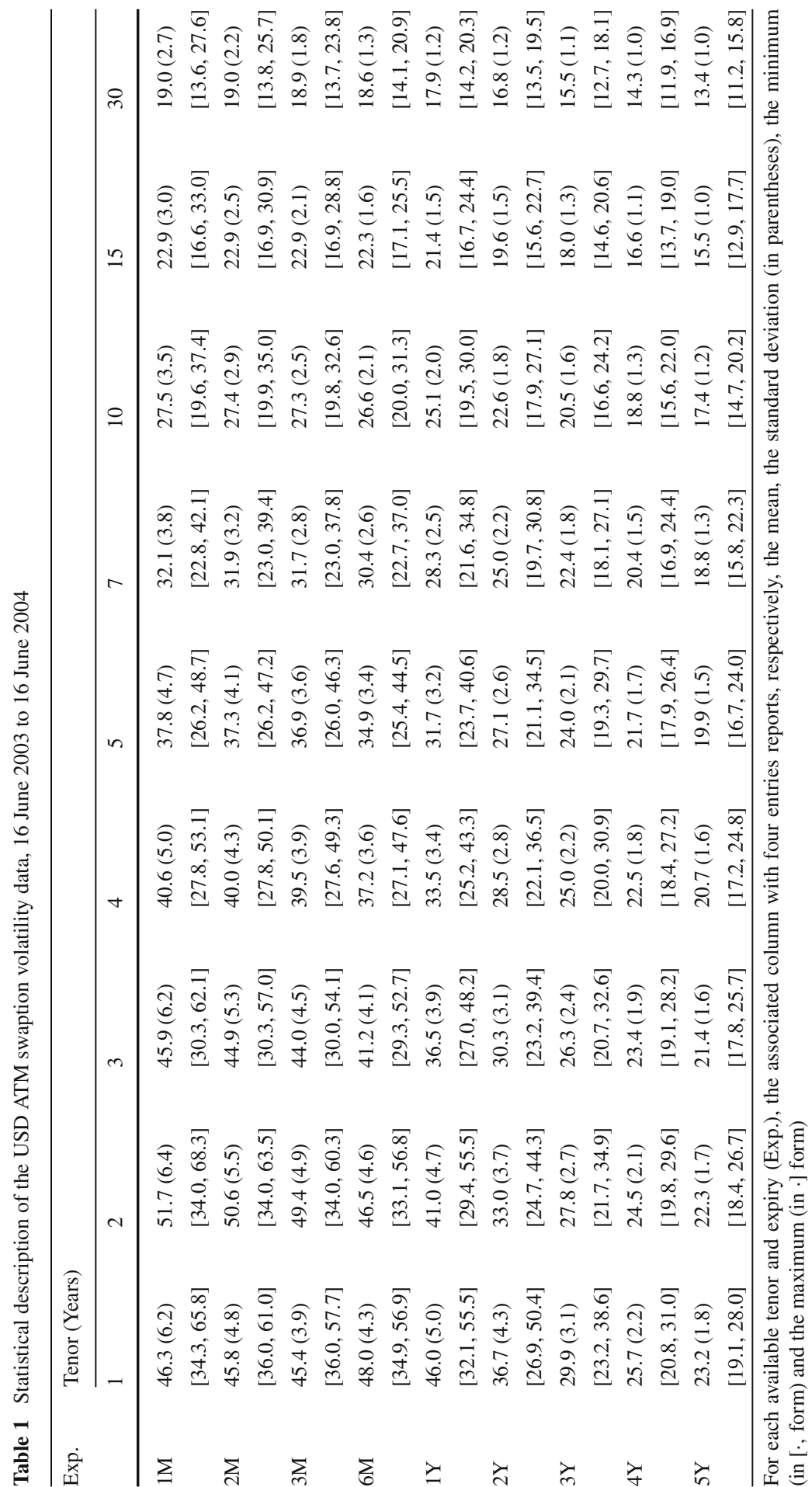


Table 2 Discount factors for the USD data of 21 February 2003

\begin{tabular}{llllll}
\hline $1 \mathrm{Y}$ & $2 \mathrm{Y}$ & $3 \mathrm{Y}$ & $4 \mathrm{Y}$ & $5 \mathrm{Y}$ & $6 \mathrm{Y}$ \\
\hline 0.98585 & 0.96223 & 0.92697 & 0.88571 & 0.84286 & 0.79986 \\
\hline
\end{tabular}

Table 3 Swaption volatility, in percentages, against strike and expiry for the USD data of 21 February 2003

\begin{tabular}{llllllllll}
\hline Exp. & \multicolumn{7}{l}{ Strike, in offset in basis points from the ATM forward swap rate } \\
\cline { 2 - 10 } & -300 & -200 & -100 & -50 & 0 & 50 & 100 & 200 & 300 \\
\hline $1 \mathrm{Y}$ & 58.78 & 45.41 & 37.34 & 35.19 & 33.15 & 32.55 & 31.99 & 31.32 & 31.21 \\
$2 \mathrm{Y}$ & 43.65 & 38.62 & 32.57 & 30.82 & 29.13 & 28.59 & 28.10 & 27.46 & 27.30 \\
$3 \mathrm{Y}$ & 40.72 & 35.12 & 30.01 & 28.46 & 26.95 & 26.12 & 25.31 & 25.03 & 24.75 \\
$4 \mathrm{Y}$ & 38.65 & 32.41 & 27.96 & 26.59 & 25.23 & 24.75 & 24.31 & 23.72 & 23.52 \\
$5 \mathrm{Y}$ & 37.17 & 30.92 & 26.66 & 25.36 & 24.08 & 23.63 & 23.20 & 22.63 & 22.43 \\
\hline
\end{tabular}

All displayed swaptions co-terminate 6 years from today. Here 'Exp.' denotes Expiry

Table 4 Error analysis of the terminal correlation measured in the Markov-functional model versus given by the approximate formula $\rho\left(\ln S_{i: n}\left(t_{i}\right), \ln S_{j: n}\left(t_{j}\right)\right) \approx \rho\left(x\left(t_{i}\right), x\left(t_{j}\right)\right)$, for a 40 years annual-paying deal, thus for a $40 \times 40$ correlation matrix

\begin{tabular}{lllll}
\hline M.r. (\%) & Max. abs. err. & Max. rel. err. (\%) & Avg. abs. err. & Avg. rel. err. (\%) \\
\hline 0 & $1.6 \times 10^{-4}$ & 0.0190 & $4.5 \times 10^{-5}$ & 0.0076 \\
5 & $5.0 \times 10^{-5}$ & 0.0072 & $1.2 \times 10^{-5}$ & 0.0030 \\
10 & $2.1 \times 10^{-5}$ & 0.0032 & $3.0 \times 10^{-6}$ & 0.0012 \\
15 & $1.0 \times 10^{-5}$ & 0.0018 & $9.8 \times 10^{-7}$ & 0.0006 \\
20 & $5.7 \times 10^{-6}$ & 0.0011 & $4.0 \times 10^{-7}$ & 0.0003 \\
\hline
\end{tabular}

m.r. mean reversion, max. maximum, abs. absolute, err. error, rel. relative, and avg. average

$14 \%$. The test is performed at various mean reversion levels, $0,5,10,15$, and $20 \%$. The terminal correlation matrix within the Markov-functional model is calculated numerically on a lattice under the terminal measure and subsequently compared to the correlation matrix given by the approximate formula (3). Note that the comparison contains two sources of error: First, the approximation (3), and, second, the numerical error inherent in the lattice calculation. In Table 4, various descriptive data for the comparison test have been displayed. Reported are, over the entries in the matrix, the maximum absolute and relative errors, and the average absolute and relative errors. As can be seen from Table 4, these errors are quite small, especially considered over a 40 years horizon.

\section{Empirical comparison results}

In this section, we report the results of our empirical comparison. The deal description is given in Table 5. For market models we use the terminal measure, 10,000 simulation 
Table 5 The Bermudan swaption deal used in the comparison

\begin{tabular}{ll}
\hline Trade: & Bermudan swaption \\
Trade type: & Receive fixed \\
Notional: & USD $100 \mathrm{~m}$ \\
Start date: & $16-J u n-2004$ \\
End date: & $16-J u n-2014$ \\
Fixed rate: & $3.2 \%$ \\
Index coupon: & Per annum \\
Index basis: & ACT/365 \\
Roll type: & Modified following \\
Callable: & At fixing dates \\
\hline
\end{tabular}

paths (5,000 plus 5,000 antithetic) and 10 stochastic factors (a full factor model), bar when $a=0 \%$, we use a single factor model. To determine the exercise boundary in market models, we use the least-squares Monte Carlo algorithm of Longstaff and Schwartz (2001), with all forward rates as explanatory variables, i.e., all available LIBOR rates for the LIBOR market model and all available swap rates for the swap market model. The reason for using all available rates as explanatory variables is that the multi factor nature of the market models needs be retained (if at all present; for $a=0 \%$ a single factor model must be used). As basis functions we use a constant and one linear term per explanatory variable, $\left\{1, X_{1}, \ldots, X_{m}\right\}$, where $m$ denotes the number of explanatory variables. The NPVs, deltas and vegas of the deal are calculated at each trade date from 16 June 2003 till 15 June 2004, inclusive, for the mean reversion levels 0, 5 and 10\%. A price comparison has been displayed in Fig. 2. As can be seen from the figure, the Markov-functional and market models are similar in terms of NPV, and prices co-move and stay together over time.

The models are, more importantly, compared in terms of hedge performance. With respect to hedging, we use so-called bucket hedging rather than factor hedging. With factor hedging, the number of hedge instruments equals the number of factors in the model. Risk sensitivities are calculated by perturbing only the model intrinsic factors. With bucket hedging, the number of hedge instruments equals the number of market traded instruments to which the model has been calibrated to. Risk sensitivities are calculated by perturbing the value of a market traded asset, and then by re-valuation of the derivative in a model re-calibrated to the perturbed market data. The reasons that we employ bucket hedging rather than factor hedging are twofold. First, Driessen et al. (2003, Section 7.3) show that bucket hedging outperforms factor hedging for caps and European swaptions (for delta hedging). Second, bucket hedging corresponds to financial practice.

Two types of hedges are considered:

(1) Delta hedging only.

(2) Delta and vega hedging.

The delta hedge is set up in terms of discount bonds, one discount bond for each tenor time associated with the deal. In the case of the deal of Table 5, there are 11 such discount bonds. To set up a joint delta and vega hedge, we proceed in the following 


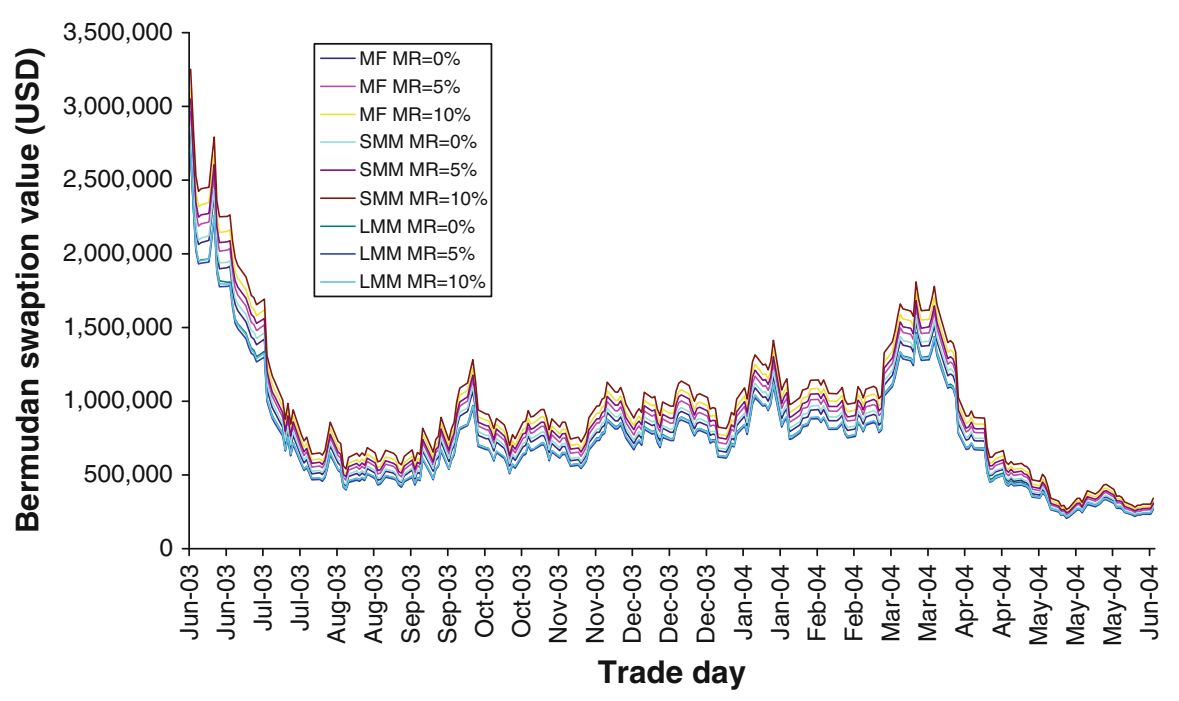

Fig. 2 Bermudan swaption values per trade date in the period 16 June 2003 to 16 June 2004, for the swap Markov-functional model, and for the LIBOR and swap market models, for correlation specifications 0, 5 and $10 \%$

four steps. First, we calculate the vegas of the 10 underlying European swaptions. Second, we calculate the amount of each of the European swaptions needed to have zero portfolio vega for all underlying volatilities. Third, the aggregate delta position, of the Bermudan and European swaptions, is calculated. Fourth, discount bonds are acquired to obtain zero delta exposure for all 11 delta buckets.

The risk sensitivities are calculated in two ways, as detailed in Sect. 2.3, (1) finite differences with perturbation sizes $1 \mathrm{bp}$ for delta and $5 \mathrm{bp}$ for vega (referred to as 'large' perturbation sizes), and, (2) constant exercise method, with perturbation sizes $10^{-5}$ bp for both delta and vega (referred to as 'small' perturbation sizes).

We note here that the computational time of calculating the NPV, the 11 deltas and the 10 vegas, at any particular trade date, is around $92 \mathrm{~s}$ for market models ${ }^{4}$ with ordinary LS, around $42 \mathrm{~s}$ for market models with constant exercise method, versus $3 \mathrm{~s}$ for the Markov-functional model. This difference of computational time is inherent to the (least squares) Monte Carlo implementation of market models versus the lattice implementation of Markov-functional models. Of course, such lattice implementation is allowed only because of the mild path-dependency of Bermudan swaptions.

The hedge portfolios are set up at each trade day and the change in portfolio value on the next trade day is recorded. The hedge test results are ordered in three subsections.

\footnotetext{
4 There are fast algorithms for implementation of market models with Monte Carlo, see Joshi (2003) for LIBOR models, and Pietersz and van Regenmortel (2006, Section 5 ) for swap models. Needless to say, we used these fast algorithms.
} 


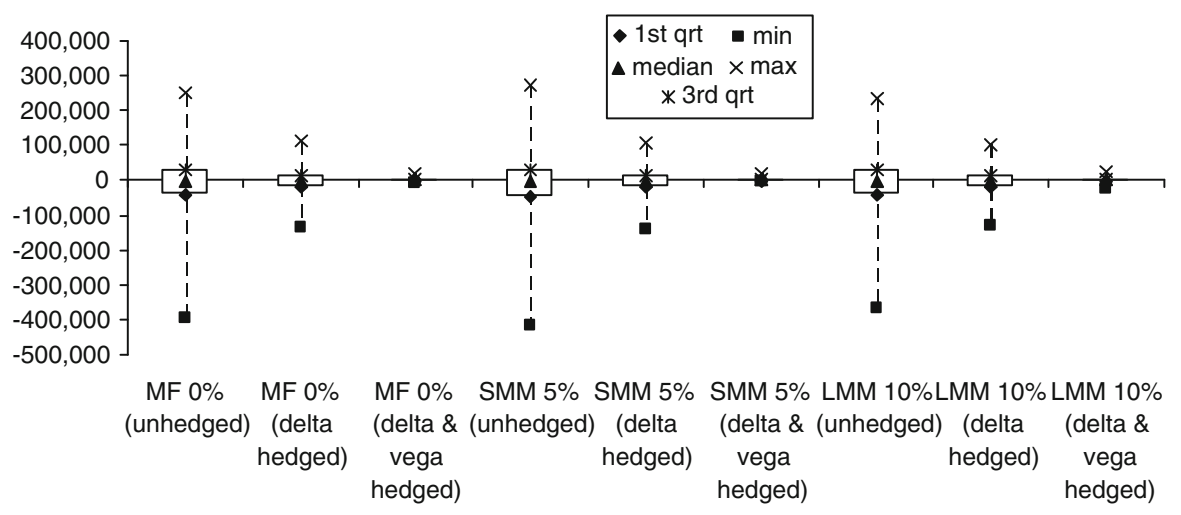

Fig. 3 Comparison of delta versus delta and vega hedging. Box-whisker plots for the change in value (in USD) of the hedged portfolio. The percentages denote the mean reversion level (MF) or correlation parametrization parameter (LMM and SMM). For market models, we use the constant exercise method, with 'small' perturbation sizes

\subsection{Delta hedging versus delta and vega hedging}

The performance of delta hedging versus delta and vega hedging is compared. Boxwhisker plots, for the change in hedge portfolio value, have been displayed in Fig. 3, for various models and mean reversion or correlation parametrization parameters. Here, MF, LMM, and SMM denote, respectively, Markov-functional model, LIBOR market model and swap market model. Box-whisker plots provide a convenient representation of a distribution, by displaying five of its key characteristics: the minimum, median, and maximum values, and the first and third quartiles.

We draw the following conclusions from the box-whisker plots in Fig. 3:

1. Delta hedging significantly decreases variance of P\&L.

2. Vega hedging additional to delta hedging significantly further decreases variance of P\&L.

It is clear that a joint delta and vega hedge by far outperforms a delta hedge. Therefore we omit, in the remainder of the paper, further study of delta hedges without a vega hedge.

\section{2 "Large" perturbation sizes versus constant exercise method with "small" perturbation sizes}

The performance of joint delta-vega hedging is compared as dependent on the method used to calculate risk sensitivities. Box-whisker plots for the change in value of the delta-vega hedged portfolios, with a mean reversion of $0 \%$ or a correlation parameter of $0 \%$, have been displayed in Fig. 4. Here, 'const. ex.' and 'pert.' denote 'constant exercise method' and 'perturbation', respectively. The analogous box-whisker plots for mean reversion or correlation parameters 5 and $10 \%$ are similar. We draw the following conclusions from the box-whisker plots in Fig. 4. 


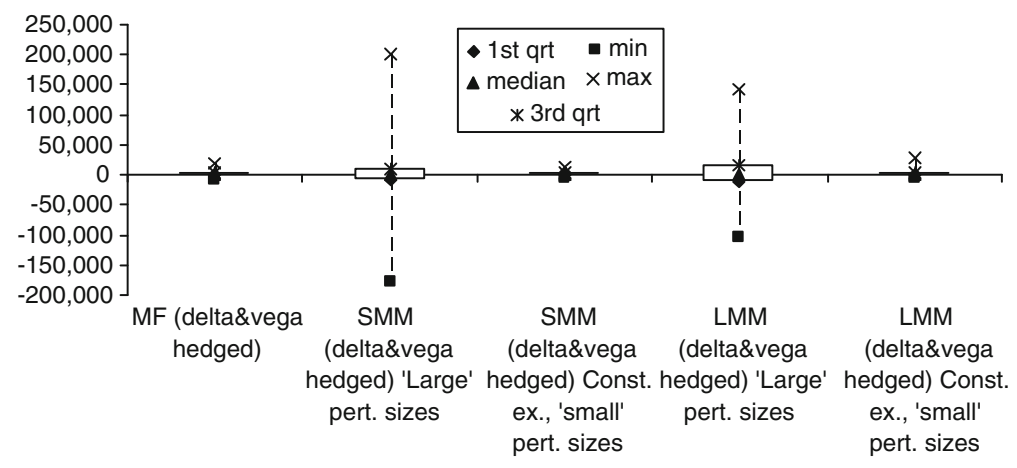

Fig. 4 'Large' perturbation sizes versus constant exercise method with 'small' perturbation sizes. Boxwhisker plots for the change in value (in USD) of the hedged portfolio. Mean reversion or correlation parameter of $0 \%$

1. The estimation of sensitivities by finite differences over MC with 'large' perturbation sizes adversely affects the variance of $\mathrm{P} \& \mathrm{~L}$ for hedging in market models.

2. The best performing Greek calculation method, for delta-vega hedging, is the constant exercise method, for which we approximately obtain similar results as with the Markov-functional model.

3. The use of the constant exercise method enables proper functioning of market models as risk management tools, for callable products on underlying assets that are continuously dependent on initial market data.

It is clear that the constant exercise method with 'small' perturbation sizes by far outperforms ordinary LS with 'large' perturbation sizes. The theoretical explanation of this out-performance is related to two issues. First, the classical LS algorithm causes a discontinuity in the numeraire relative payoff along the path, which renders finite difference estimates of sensitivities to be less efficient. Second, 'larger' perturbation sizes cause more variance in the finite difference estimate of a sensitivity, since the correlation between the payoff in the original and perturbed models becomes smaller. These two effects lead to more Monte Carlo caused randomness in the contents of the hedge portfolio, which ultimately leads to increased variance of P\&L, as can be seen in Fig. 4.

We omit, in the remainder of the paper, further study of ordinary LS with 'large' perturbation sizes.

\subsection{Delta-vega hedge results}

The performance of joint delta-vega hedging is compared across models and mean reversion or correlation specifications. For the market models, we use the constant exercise method with 'small' perturbation sizes. Box-whisker plots for the change in value of the delta-vega hedged portfolios have been displayed in Fig. 5. Numerical details for Fig. 5 may be found in Appendix 1. We draw the following conclusions from the box-whisker plots in Fig. 5. 


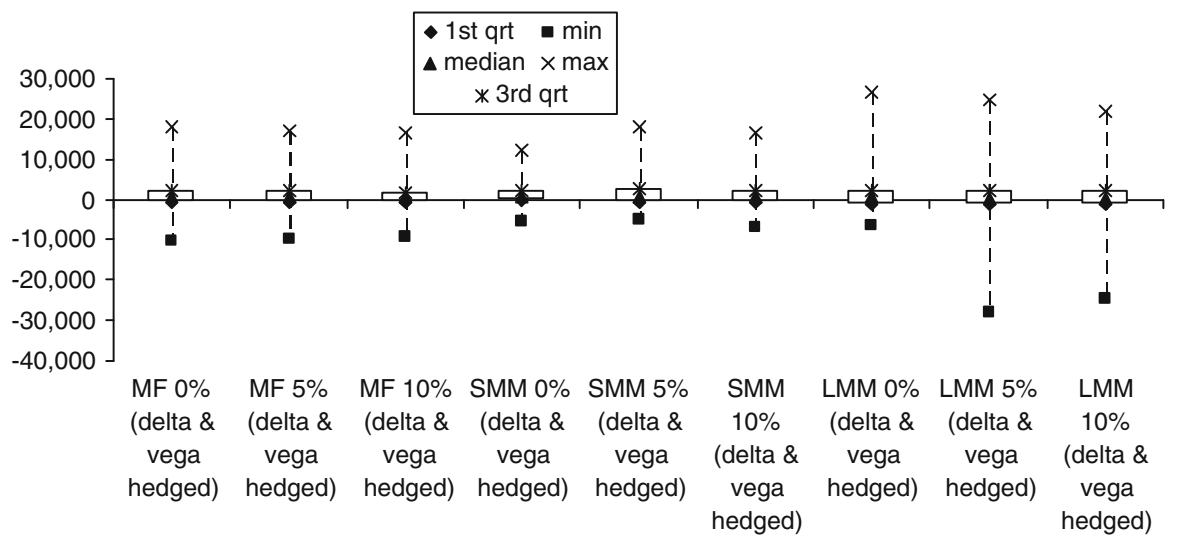

Fig. 5 Delta-vega hedge results. Box-whisker plots for the change in value (in USD) of the hedged portfolio. The percentages denote the mean reversion level (MF) or correlation parametrization parameter (LMM and SMM). For market models, we use the constant exercise method, with 'small' perturbation sizes

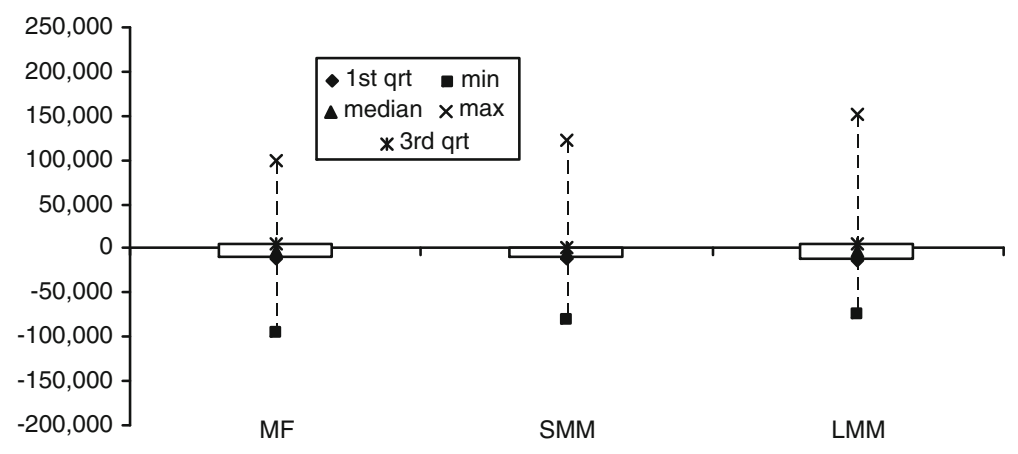

Fig. 6 Monthly hedge results. Box-whisker plots for the change in value (in USD) of the hedged portfolio. Mean reversion or correlation parameter of $5 \%$

1. The impact of mean reversion or correlation parameter specification on hedge performance is not very large.

2. The hedge performance for all three models is very similar.

\subsection{Impact of monthly hedging}

The hedge studies in Sects. 5.1-5.3 are based on rebalancing the hedge portfolio every trade day. In this section, we assess the impact of rebalancing at monthly intervals. We extended the data set to include the period from 9 February 1999 to 16 June 2003. In total, there are 65 trade days separated by monthly intervals. Moving from approximately 1 day intervals to approximately 30 days intervals, we expect an increase in variance of profit and loss of about $\sqrt{30} \approx 5.5$. The results for monthly hedging are displayed in Fig. 6. Indeed, the scale of Fig. 6 relates to the scale of Fig. 5 (daily hedging) as approximately 5.5 to 1 . 


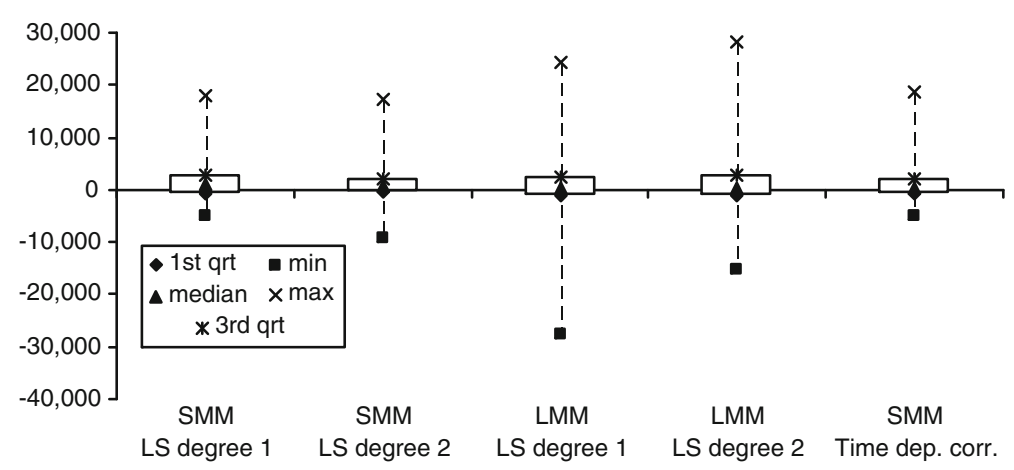

Fig. 7 Degree 1 versus degree 2 basis functions (left four plots) and time dependent versus constant correlation (right most plot). Box-whisker plots for the change in value (in USD) of the hedged portfolio. Correlation parameter of $5 \%$

\subsection{Higher order basis functions for least-squares MC}

We investigate the impact of higher order polynomials for least-squares Monte Carlo simulation. The basis functions used in Sects. 5.1-5.4 are a constant and one linear term per explanatory variable, $\left\{1, X_{1}, \ldots, X_{m}\right\}$, which we refer to as "degree 1 " basis functions. The set of "degree 2" basis functions consists of degree 1 basis functions plus functions $X_{i} X_{j}$ for $1 \leq i \leq j \leq m$. Note that quadratic functions $X_{i}^{2}$ are included in this definition. The hedge results for degree 1 versus degree 2 basis functions are displayed in the left four box-whisker plots of Fig. 7. The results show that including higher order basis functions does not have a significant effect on the resulting reduction of variance of P\&L.

\subsection{Time varying correlation}

For the hedge tests in Sects. 5.1-5.5 we use constant correlation in market models as specified in (1). Several authors (e.g., Andersen and Brotherton-Ratcliffe 2005; Li and Zhao 2006) consider time varying correlation. To assess the impact of time varying correlation, we use a two-factor parametrization due to Andersen and BrothertonRatcliffe (2005, Section 5.2):

$$
\rho_{i j}(t)=\frac{\lambda_{i}(t) \cdot \lambda_{j}(t)}{\sqrt{\left\|\lambda_{i}(t)\right\|\left\|\lambda_{j}(t)\right\|}},
$$

with the two-dimensional vector $\lambda_{i}(t)$ given by:

$$
\lambda_{i}(t)=\left(\gamma_{11}+\gamma_{12} e^{-\gamma_{13}\left(t_{i}-t\right)}, \gamma_{21}+\gamma_{22} e^{-\gamma_{23}\left(t_{i}-t\right)}\right) .
$$

We use the same parameters as Andersen and Brotherton-Ratcliffe (2005): $\gamma_{11}=$ $1.5 \%, \gamma_{12}=2.5 \%, \gamma_{13}=5 \%, \gamma_{21}=1 \%, \gamma_{22}=-5 \%, \gamma_{23}=10 \%$. Given the lack of sensitivity to correlation in the hedge results of Fig. 5 we expect little impact from time 
varying correlation. The hedge results with time varying correlation are displayed in the right most box-whisker plot of Fig. 7. The results show that time dependent correlation indeed has an insignificant impact on the reduction of variance of P\&L.

\section{The impact of smile}

In this section, we provide details on how the Markov-functional and swap market models can be fitted to smile and investigate the impact of smile relative to the impact of correlation cq. mean reversion on the prices of Bermudan swaptions. As a concrete example, the displaced diffusion smile dynamics of Rubinstein (1983) are considered. In a displaced diffusion setting, the forward swap rate is modelled as

$$
S_{i: n}(t)=\tilde{S}_{i: n}(t)-r_{i}, \frac{\mathrm{d} \tilde{S}_{i: n}(t)}{\tilde{S}_{i: n}(t)}=\sigma_{i: n} \mathrm{~d} W^{(i: n)}(t),
$$

with $r_{i}$ the displacement parameter and $W^{(i: n)}$ a Brownian motion under the forward swap measure associated with $S_{i: n}$. The solution to stochastic differential equation (SDE) (8) is

$$
S_{i: n}(t)=-r_{i}+\left(S_{i: n}(0)+r_{i}\right) \exp \left\{\sigma_{i: n} W^{(i: n)}(t)-\frac{1}{2} \sigma_{i: n}^{2} t\right\} .
$$

The displaced diffusion extension is first discussed for the Markov-functional model and second for the swap market model. The Markov-functional model is fitted to volatility by fitting the digital swaptions. The value $V^{(i)}$ of the digital swaption on swap rate $S_{i: n}\left(t_{i}\right)$ with strike $K$ is given by the familiar formula in the Black world

$$
V^{(i)}=\operatorname{PVBP}_{i: n}(0) N\left(d_{2}^{(i)}\right), \quad d_{2}^{(i)}=\frac{\log \left(K / S_{i: n}(0)\right)-\frac{1}{2} \sigma_{i: n}^{2} t_{i}}{\sigma_{i: n} \sqrt{t_{i}}}
$$

where $N(\cdot)$ denotes the cumulative normal distribution function and where $\mathrm{PVBP}_{i: n}$ denotes the present value of a basis point, $\mathrm{PVBP}_{i: n}=\sum_{k=i}^{n} \alpha_{i} B_{i+1}(t)$. Here $\alpha_{i}$ denotes the day count fraction for period $\left[t_{i}, t_{i+1}\right]$ and $B_{i}(t)$ denotes the time- $t$ value of a discount bond for payment of one unit of currency at time $t_{i}$. In the displaced diffusion world, the value $\tilde{V}^{(i)}$ of the digital swaption is given by a displaced forward swap rate and strike

$$
\tilde{V}^{(i)}=\operatorname{PVBP}_{i: n}(0) N\left(\tilde{d}_{2}^{(i)}\right), \quad \tilde{d}_{2}^{(i)}=\frac{\log \left(\frac{S_{i: n}(0)+r_{i}}{K+r_{i}}\right)-\frac{1}{2} \sigma_{i: n}^{2} t_{i}}{\sigma_{i: n} \sqrt{t_{i}}} .
$$

The implementation of a non-smile Markov-functional model has to be changed only in two places to incorporate displaced diffusion smile dynamics. First, the functional form of the terminal discount bond $B_{n+1}$ at time $t_{n}$ is determined, using the equation 


$$
B_{n+1}\left(t_{n}\right)=\frac{1}{1+\alpha_{n} S_{n: n}\left(t_{n}\right)}
$$

In a non-smile Markov-functional model, we then have

$$
B_{n+1}\left(t_{n}, x\left(t_{n}\right)\right)=\frac{1}{1+\alpha_{n} S_{n: n}(0) \exp \left\{-\frac{1}{2} \sigma_{n: n}^{2} t_{n}+\frac{\sigma_{n: n}}{e^{2 a t_{n}}-1} x\left(t_{n}\right)\right\}},
$$

this is exactly the penultimate equation on page 399 of Hunt et al. (2000). In a displaced diffusion setting, we substitute (9) into (12) and then (13) becomes

$$
\tilde{B}_{n+1}\left(t_{n}, x\left(t_{n}\right)\right)=\frac{1}{1+\alpha_{n}\left[-r_{i}+\left(S_{n: n}(0)+r_{i}\right) \exp \left\{-\frac{1}{2} \sigma_{n: n}^{2} t_{n}+\frac{\sigma_{n: n}}{e^{2 a t_{n}}-1} x\left(t_{n}\right)\right\}\right]},
$$

Second, the functional forms of the swap rates $S_{i: n}\left(t_{i}, \cdot\right), i=1, \ldots, n-1$ are determined, by inverting the value of the digital swaption against strike. In a non-smile Markov-functional model, we invert (10) and obtain

$$
S_{i: n}\left(t_{i}, x\left(t_{i}\right)\right)=S_{i: n}(0) \exp \left\{-\frac{1}{2} \sigma_{i: n}^{2} t_{i}-\sigma_{i: n} \sqrt{t_{i}} N^{-1}\left(\frac{J^{(i)}\left(x\left(t_{i}\right)\right)}{\operatorname{PVBP}_{i: n}(0)}\right)\right\}
$$

with $J^{(i)}(x)$ denoting the value of a digital swaption with strike $x$ in the model, calculated by induction from $i=n-1, \ldots, 1$. In a displaced diffusion setting, we invert (11) to obtain

$S_{i: n}\left(t_{i}, x\left(t_{i}\right)\right)=-r_{i}+\left(S_{i: n}(0)+r_{i}\right) \exp \left\{-\frac{1}{2} \sigma_{i: n}^{2} t_{i}-\sigma_{i: n} \sqrt{t_{i}} N^{-1}\left(\frac{J^{(i)}\left(x\left(t_{i}\right)\right)}{\mathrm{PVBP}_{i: n}(0)}\right)\right\}$

Next, the displaced diffusion swap market model is made reference to. The dynamics of the forward swap rates under the terminal measure in general smile models can be found in Jamshidian (1997, Eq. (6), p. 320).

We fit the displaced diffusion model to the market data of Table 3 and find the volatility parameters $\sigma_{i: n}$ and displacement parameters $r_{i}$ as listed in Table 6 . The fitted volatility and fit errors have been displayed in Table 7. As can be seen from the table, the displaced diffusion model fits the market well for ATM and out-of-the-money (OTM) options (fit error less than a percent), but not so well for in-the-money (ITM) options, for which the model underfits the market up to $21 \%$. We note here that the disability of obtaining a perfect fit to the smile volatility data is due solely to the displaced diffusion model, and not to the Markov-functional or market models. An exact fit to the swaption smile surface can be obtained, for example, with the relative-entropy minimization framework of Avellaneda et al. (1997). To benchmark the implementation of the displaced diffusion Markov-functional and swap market models, European swaptions are valued in (1) a constant volatility model with the volatility associated with the expiry and strike of the swaption and (2) the smile model. The results of this test for the Markov-functional model have been displayed in Table 8. The benchmark 
Table 6 Volatility $\sigma_{i: n}$ and displaced diffusion $r_{i}$ parameters fitted to USD market data of 21 February 2003

\begin{tabular}{llllll}
\hline$i$ & 1 & 2 & 3 & 4 & 5 \\
\hline Expiry & $1 \mathrm{Y}$ & $2 \mathrm{Y}$ & $3 \mathrm{Y}$ & $4 \mathrm{Y}$ & $5 \mathrm{Y}$ \\
Tenor & $5 \mathrm{Y}$ & $4 \mathrm{Y}$ & $3 \mathrm{Y}$ & $2 \mathrm{Y}$ & $1 \mathrm{Y}$ \\
$\sigma_{i: n}$ & $28.29 \%$ & $21.76 \%$ & $18.28 \%$ & $16.08 \%$ & $14.62 \%$ \\
$r_{i}$ & $0.71 \%$ & $1.55 \%$ & $2.33 \%$ & $2.89 \%$ & $3.39 \%$ \\
\hline
\end{tabular}

Table 7 Fitted swaption volatility and fit errors with the displaced diffusion model, in percentages, against strike and expiry for the USD data of 21 February 2003

\begin{tabular}{llllllllll}
\hline Exp. & \multicolumn{7}{l}{ Strike, in offset in basis points from the ATM forward swap rate } \\
\cline { 2 - 11 } & -300 & -200 & -100 & -50 & 0 & 50 & 100 & 200 & 300 \\
\hline Fitted swaption volatility & & & & & & & \\
$1 \mathrm{Y}$ & 37.82 & 35.11 & 33.88 & 33.48 & 33.15 & 32.89 & 32.66 & 32.30 & 32.03 \\
$2 \mathrm{Y}$ & 34.32 & 31.57 & 30.07 & 29.54 & 29.11 & 28.74 & 28.43 & 27.92 & 27.51 \\
$3 \mathrm{Y}$ & 32.23 & 29.58 & 28.02 & 27.45 & 26.98 & 26.57 & 26.21 & 25.63 & 25.16 \\
$4 \mathrm{Y}$ & 30.34 & 27.83 & 26.29 & 25.72 & 25.23 & 24.82 & 24.46 & 23.85 & 23.37 \\
$5 \mathrm{Y}$ & 29.17 & 26.74 & 25.21 & 24.63 & 24.14 & 23.72 & 23.35 & 22.73 & 22.23 \\
Absolute fit errors, & model volatility minus & market volatility & & & & \\
$1 \mathrm{Y}$ & -20.96 & -10.29 & -3.46 & -1.71 & 0.00 & 0.34 & 0.67 & 0.98 & 0.82 \\
$2 \mathrm{Y}$ & -9.33 & -7.05 & -2.50 & -1.28 & -0.02 & 0.15 & 0.33 & 0.45 & 0.21 \\
$3 \mathrm{Y}$ & -8.49 & -5.54 & -1.99 & -1.01 & 0.02 & 0.44 & 0.90 & 0.60 & 0.41 \\
$4 \mathrm{Y}$ & -8.31 & -4.58 & -1.67 & -0.87 & 0.00 & 0.06 & 0.14 & 0.13 & -0.15 \\
$5 \mathrm{Y}$ & -8.00 & -4.18 & -1.45 & -0.73 & 0.06 & 0.09 & 0.14 & 0.10 & -0.20 \\
\hline
\end{tabular}

All displayed swaptions co-terminate 6 years from today. Here 'Exp.' denotes Expiry

is of high quality, though there are some slight differences due to numerical errors in the grid calculation. The benchmark results for the swap market model are of similar good quality.

Subsequently, Bermudan swaptions are priced with varying strikes and otherwise specified in Table 9. The Bermudan swaptions are priced in the Markov-functional and SMM models, and in their displaced diffusion counterparts, at various mean reversion or correlation parameter levels. In the non-smile models, there are two possibilities for choosing the volatilities. First, the volatilities can be used that correspond to the strike of the Bermudan swaption. Second, the ATM volatilities can be used, regardless of the strike of the Bermudan swaption. The calculated prices have been displayed in Table 10. The results in the table show that the impact of correlation is significant, since a $10 \%$ change in mean reversion can cause a change in value equal to a parallel volatility shift of $1 \%$. The impact of correlation is comparable to that reported by Choy et al. (2004, Table 11 ), though the latter authors name this impact 'non-substantial'. The impact of smile is, for the deal considered, much larger than the impact of correlation and mean reversion, since $10 \%$ mean reversion is usually a high level when observed in the market. In terms of vega, the smile impact can be as large as a parallel 
Table 8 Benchmark results for the displaced diffusion Markov-functional model: European swaption prices in a constant volatility model versus a smile model

\begin{tabular}{|c|c|c|c|c|c|c|c|c|c|}
\hline \multirow[t]{2}{*}{ Exp. } & \multicolumn{9}{|c|}{ Strike, in offset in basis points from the ATM forward swap rate } \\
\hline & -300 & -200 & -100 & -50 & 0 & 50 & 100 & 200 & 300 \\
\hline \multicolumn{10}{|c|}{ Constant volatility Markov-functional model } \\
\hline $1 \mathrm{Y}$ & 328 & 52,561 & & & $2,340,174$ & $3,689,485$ & $5,298,889$ & $9,053,757$ & $13,203,090$ \\
\hline $2 Y$ & 24,540 & 249,639 & $1,018,213$ & $1,683,785$ & $2,542,759$ & $3,580,442$ & $4,773,685$ & $7,521,975$ & $10,595,480$ \\
\hline $3 \mathrm{Y}$ & 84,026 & 391,072 & $1,098,965$ & $1,629,508$ & $2,276,285$ & $3,030,862$ & $3,881,504$ & $5,818,070$ & $7,984,799$ \\
\hline $4 \mathrm{Y}$ & 106,959 & 367,4 & & $1,237,1$ & 1,663 & 2,15 & 2,697 & 3,928 & $5,305,394$ \\
\hline $5 \mathrm{Y}$ & 82,725 & & & & & & & & $2,650,706$ \\
\hline \multicolumn{10}{|c|}{ Displaced diffusion Markov-functional model } \\
\hline $1 \mathrm{Y}$ & 322 & 52,255 & 605,446 & $1,303,083$ & $2,338,220$ & $3,687,599$ & $5,297,237$ & $9,052,727$ & $13,202,556$ \\
\hline $2 \mathrm{Y}$ & 24,201 & 248,060 & $1,015,124$ & $1,680,221$ & $2,538,993$ & $3,576,743$ & $4,770,238$ & $7,519,330$ & $10,593,675$ \\
\hline $3 Y$ & 83,169 & 388,983 & $1,095,781$ & $1,626,018$ & $2,272,668$ & $3,027,290$ & $3,878,101$ & $5,815,249$ & $7,982,667$ \\
\hline $4 Y$ & 105,990 & 365,588 & 875,586 & $1,234,362$ & $1,660,433$ & $2,149,153$ & $2,694,366$ & $3,926,683$ & $5,303,550$ \\
\hline $5 \mathrm{Y}$ & 82,154 & 234,125 & 502,792 & 683,627 & 894,329 & $1,132,903$ & $1,396,815$ & $1,989,378$ & $2,649,787$ \\
\hline
\end{tabular}

The notional is USD 100 million. All displayed swaptions co-terminate 6 years from today. Here 'Exp.' denotes Expiry

Table 9 The Bermudan swaption deal used in the test of impact of smile

\begin{tabular}{ll}
\hline Trade: & Bermudan swaption \\
Trade type: & Receive fixed \\
Notional: & USD $100 \mathrm{~m}$ \\
Valuation date: & $21-\mathrm{Feb}-2003$ \\
Start date: & $21-\mathrm{Feb}-2004$ \\
End date: & $21-\mathrm{Feb}-2009$ \\
Index coupon: & Per annum \\
Index basis: & ACT/365 \\
Roll type: & Modified following \\
Callable: & At fixing dates \\
\hline
\end{tabular}

shift in volatility of -8 to $1 \%$, for per-strike volatilities, and -1 to $6 \%$, for ATM volatilities. Furthermore, the displaced diffusion smile model underfitted the volatility smile observed in the market. Since increasing the volatility usually leads to a higher value for Bermudan swaptions, ${ }^{5}$ the impact of smile can thus be even higher, when ATM volatilities are used.

\section{Conclusions}

We investigated the impact of correlation on the pricing and hedge performance of Bermudan swaptions for various models. We showed how the Markov-functional

\footnotetext{
5 Pietersz and Pelsser (2004, Appendix) explain that Bermudan swaptions can in certain particular circumstances have negative vega.
} 
Table 10 Prices of Bermudan swaptions in smile versus non-smile models with various correlation/mean reversion assumptions

\begin{tabular}{|c|c|c|c|c|c|}
\hline & \multicolumn{5}{|l|}{ Strike } \\
\hline & $2 \%$ & $3 \%$ & $4 \%$ & $5 \%$ & $6 \%$ \\
\hline MF-MR=0\% & 420,954 & $1,072,043$ & $2,452,060$ & $5,047,951$ & $8,573,535$ \\
\hline Vega $1 \% \mathrm{MF}$ & 34,609 & 68,118 & 96,242 & 93,513 & 68,477 \\
\hline SMM- $a=0 \%$ & 407,667 & $1,053,210$ & $2,443,332$ & $5,065,794$ & $8,605,508$ \\
\hline SMM SE- $a=0 \%$ & 7,551 & 12,964 & 17,204 & 16,032 & 10,625 \\
\hline Vega $1 \%$ SMM & 33,105 & 66,154 & 97,141 & 91,850 & 67,194 \\
\hline MF-MR=5\% & 436,518 & $1,103,269$ & $2,495,689$ & $5,090,486$ & $8,606,769$ \\
\hline Diff. in vega & 0.4 & 0.5 & 0.5 & 0.5 & 0.5 \\
\hline SMM- $a=5 \%$ & 407,922 & $1,060,731$ & $2,461,625$ & $5,101,071$ & $8,657,349$ \\
\hline SMM SE- $a=5 \%$ & 7,386 & 12,764 & 17,228 & 16,244 & 11,243 \\
\hline Diff. in vega & 0.0 & 0.1 & 0.2 & 0.4 & 0.8 \\
\hline $\mathrm{MF}-\mathrm{MR}=10 \%$ & 452,417 & $1,135,155$ & $2,540,694$ & $5,135,323$ & $8,642,685$ \\
\hline Diff. in vega & 0.9 & 0.9 & 0.9 & 0.9 & 1.0 \\
\hline SMM- $a=10 \%$ & 405,819 & $1,062,986$ & $2,485,969$ & $5,142,268$ & $8,708,570$ \\
\hline SMM SE- $a=10 \%$ & 7,202 & 12,488 & 17,090 & 16,359 & 11,873 \\
\hline Diff. in vega & -0.1 & 0.1 & 0.4 & 0.8 & 1.5 \\
\hline Smile MF & 148,130 & 747,270 & $2,347,664$ & $5,074,574$ & $8,623,356$ \\
\hline Diff. in vega & -7.9 & -4.8 & -1.1 & 0.3 & 0.7 \\
\hline Smile SMM & 146,223 & 756,925 & $2,373,545$ & $5,094,288$ & $8,642,666$ \\
\hline Smile SMM SE & 4,710 & 11,138 & 16,781 & 14,437 & 9,801 \\
\hline Diff. in vega & -8.3 & -4.8 & -0.8 & 0.5 & 1.0 \\
\hline \multicolumn{6}{|l|}{ ATM volatilities } \\
\hline MF-MR $=0 \%$ & 67,210 & 650,483 & $2,328,235$ & $5,124,154$ & $8,691,466$ \\
\hline Vega $1 \% \mathrm{MF}$ & 14,997 & 60,797 & 95,139 & 93,681 & 72,106 \\
\hline Smile MF & 148,130 & 747,270 & $2,347,664$ & $5,074,574$ & $8,623,356$ \\
\hline Diff. in vega & 5.4 & 1.6 & 0.2 & -0.5 & -0.9 \\
\hline SMM- $a=0 \%$ & 61,944 & 610,939 & $2,286,869$ & $5,139,345$ & $8,731,084$ \\
\hline SMM SE- $a=0 \%$ & 2,626 & 10,114 & 16,763 & 16,122 & 11,628 \\
\hline Vega $1 \%$ SMM & 13,915 & 59,861 & 94,495 & 91,859 & 77,375 \\
\hline Smile SMM & 146,223 & 756,925 & $2,373,545$ & $5,094,288$ & $8,642,666$ \\
\hline Diff. in vega & 6.1 & 2.4 & 0.9 & -0.5 & -1.1 \\
\hline
\end{tabular}

Here 'MF', 'MR', ' $a$ ' and 'SE' denote 'Markov-functional model', 'mean reversion', 'the correlation parameter $a$ of (1)' and the 'standard error', respectively. Any difference ('Diff.') is with respect to a price at zero mean reversion or at zero $a$. The non-smile models use per-strike volatilities, except where indicated that ATM volatilities are used

model can approximately be fitted to terminal correlation, by developing a novel approximate formula for terminal correlation. The approximate formula was shown to be of high quality in a numerical test. Empirically, the impact of terminal correlation was shown to be somewhat significant for pricing of Bermudan swaptions in market models, and the same effect can be attained in the single-factor Markov-functional 
Table 11 Numerical details for the delta-vega hedge results

\begin{tabular}{lrrr}
\hline & MF 0\% & MF 5\% & MF 10\% \\
\hline Minimum & $-10,080$ & $-9,601$ & $-9,439$ \\
1st quartile & -702 & -569 & -485 \\
Median & 779 & 716 & 650 \\
3rd quartile & 2,217 & 2,038 & 1,888 \\
Maximum & 18,038 & 16,838 & 16,568 \\
\hline & SMM 0\% & SMM 5\% & SMM 10\% \\
\hline Minimum & $-5,702$ & $-4,871$ & $-7,120$ \\
1st quartile & -435 & -824 & -799 \\
Median & 800 & 834 & 541 \\
3rd quartile & 2,102 & 2,735 & 2,268 \\
Maximum & 12,356 & 17,855 & 16,557 \\
\hline & LMM 0\% & LMM 5\% & LMM 10\% \\
\hline 3rd quartile & 2,400 & $-27,794$ & $-24,519$ \\
Maximum & 26,721 & $-1,261$ & $-1,140$ \\
\hline Minimum & $-6,241$ & 29,497 & 485 \\
Median & $-1,057$ & 2,423 & 2,018 \\
\hline
\end{tabular}

Box-whisker tables for the change in value (in USD) of the hedged portfolio. The percentages denote the mean reversion level (MF) or correlation parametrization parameter (LMM and SMM). For market models, we use the constant exercise method, with é6smallć6 perturbation sizes

model by calibration to terminal correlation. We showed empirically by comparison with decorrelation in multi factor market models that hedge performance for Bermudan swaptions is, for practical purposes, almost identical, regardless of the model, number of factors, or correlation specification. Our results show that the need of modelling correlation can already be adequately met by a single factor model. Whether these results extend beyond the asset class of Bermudan swaptions, is an interesting question that we leave to answer in future research. With respect to hedge portfolios, we showed (1) that delta hedging significantly reduces variance of P\&L in both Markov-functional and market models, (2) that vega hedging additional to delta hedging significantly further reduces variance of $\mathrm{P} \& \mathrm{~L}$ in both Markov-functional and market models, (3) that estimation of Greeks by finite differences over Monte Carlo for callable products with the regular LS algorithm and 'large' perturbation sizes adversely affects the delta-vega hedge performance of market models. We showed the constant exercise method with 'small' perturbation sizes enables proper functioning of market models as risk management tools, for callable products on underlying assets that are continuously dependent on initial market data. Moreover, we investigated the impact of smile via displaced diffusion versions of the Markov-functional and swap market 
models. For a particular deal and USD market data, we showed that the impact of smile is much larger that the impact of correlation.

Open Access This article is distributed under the terms of the Creative Commons Attribution Noncommercial License which permits any noncommercial use, distribution, and reproduction in any medium, provided the original author(s) and source are credited.

\section{Appendix 1: Numerical details for Fig. 5}

Numerical details for Fig. 5 are given in Table 11.

\section{References}

Andersen, L., \& Andreasen, J. (2001). Factor dependence of bermudan swaptions: Fact or fiction? Journal of Financial Economics, 62(1), 3-37.

Andersen, L., \& Brotherton-Ratcliffe, R. (2005). Extended LIBOR market models with stochastic volatility. Journal of Computational Finance, 9(1), 1-40.

Avellaneda, M., \& Gamba, R. (2001). Conquering the Greeks in Monte Carlo: Efficient calculation of the market sensitivities and hedge-ratios of financial assets by direct numerical simulation. In Proceedings of the first bachelier congress, Paris.

Avellaneda, M., Holmes, R., Friedman, C., \& Samperi, D. (1997). Calibration of volatility surfaces via relative-entropy minimization. Applied Mathematical Finance, 4(1), 37-64.

Bennett, M. N., \& Kennedy, J. E. (2005). A comparison of Markov-functional and market models: The one-dimensional case. Journal of Derivatives, 13(2), 22-43.

Black, F. (1976). The pricing of commodity contracts. Journal of Financial Economics, 3(2), 167-179.

Black, F., Derman, E., \& Toy, W. (1990). A one-factor model of interest rates and its applications to treasury and bond options. Financial Analysts Journal, 46(1), 33-39.

Black, F., \& Karasinski, P. (1991). Bond and option pricing when short rates are lognormal. Financial Analysts Journal, 47(4), 52-59.

Brace, A., Gạtarek, D., \& Musiela, M. (1997). The market model of interest rate dynamics. Mathematical Finance, 7(2), 127-155.

Brigo, D. (2002). A note on correlation and rank reduction. Working paper, www.damianobrigo.it.

Choy, B., Dun, T., \& Schlögl, E. (2004). Correlating market models, Risk Magazine, 17, 124-129.

Cox, J. C., Ingersoll, J. E., \& Ross, S. A. (1985). A theory of the term structure of interest rates. Econometrica, 53(2), 385-408.

Dai, Q., \& Singleton, K. (2003). Term structure dynamics in theory and reality. Review of Financial Studies, 16(3), 631-678.

Dothan, L. U. (1978). On the term structure of interest rates. Journal of Financial Economics, 6(1), 59-69.

Driessen, J., Klaassen, P., \& Melenberg, B. (2003). The performance of multi-factor term structure models for pricing and hedging caps and swaptions. Journal of Financial and Quantitative Analysis, 38(3), 635-672.

Fan, R., Gupta, A., \& Ritchken, P. (2003). Hedging in the possible presence of unspanned stochastic volatility: Evidence from swaption markets. Journal of Finance, 58(5), 2219-2248.

Fournié, E., Lasry, J. -M., Lebuchoux, J., Lions, P. -L., \& Touzi, N. (1999). Applications of Malliavin calculus to Monte Carlo methods in finance. Finance and Stochastics, 3(4), 391-412.

Glasserman, P. (2004). Monte Carlo methods in financial engineering. Berlin: Springer.

Glasserman, P., \& Zhao, X. (1999). Fast Greeks by simulation in forward LIBOR models. Journal of Computational Finance, 3(1), 5-39.

Grubišić, I., \& Pietersz, R. (2007). Efficient rank reduction of correlation matrices. Linear Algebra and Its Applications, 422(2-3), 629-653.

Gupta, A., \& Subrahmanyam, M. (2005). Pricing and hedging interest rate options: Evidence from cap-floor markets. Journal of Banking and Finance, 29(3), 701-733.

Ho, T. S. Y., \& Lee, S. -B. (1986). Term structure movements and pricing interest rate contingent claims. Journal of Finance, 41(5), 1011-1029. 
Hull, J. C., \& White, A. (1990). Pricing interest-rate-derivative securities. Review of Financial Studies, 3(4), 573-592.

Hull, J. C., \& White, A. (2000). Forward rate volatilities, swap rate volatilities, and implementation of the LIBOR market model. Journal of Fixed Income, 10(2), 46-62.

Hunt, P., Kennedy, J., \& Pelsser, A. A. J. (2000). Markov-functional interest rate models. Finance and Stochastics, 4(4), 391-408.

Hunter, C. J., Jäckel, P., \& Joshi, M. S. (2001). Getting the drift. Risk Magazine, 14, 81-86.

Jamshidian, F. (1997). LIBOR and swap market models and measures. Finance and Stochastics, 1(4), 293-330.

Joshi, M. S. (2003). Rapid computation of drifts in a reduced factor LIBOR market model. Wilmott Magazine, 5, 84-85.

Li, H., \& Zhao, F. (2006). Unspanned stochastic volatility: Evidence from hedging interest rate derivatives. Journal of Finance, 61(1), 341-378.

Longstaff, F. A., Santa-Clara, P., \& Schwartz, E. S. (2001). Throwing away a billion dollars: The cost of suboptimal exercise strategies in the swaptions market. Journal of Financial Economics, 62(1), 39-66.

Longstaff, F. A., \& Schwartz, E. S. (2001). Valuing American options by simulation: A simple least-squares approach. Review of Financial Studies, 14(1), 113-147.

Miltersen, K. R., Sandmann, K., \& Sondermann, D. (1997). Closed form solutions for term structure derivatives with log-normal interest rates. Journal of Finance, 52(1), 409-430.

Ostrovsky, D. (2002). A Markov-functional model consistent with caplet and swaption smiles. Yale University Working Paper.

Pietersz, R., \& Groenen, P. J. F. (2004a). A major LIBOR fit. Risk Magazine, 17(December), 102.

Pietersz, R., \& Groenen, P. J. F. (2004b). Rank reduction of correlation matrices by majorization. Quantitative Finance, 4(6), 649-662.

Pietersz, R., \& Pelsser, A. A. J. (2004). Risk-managing Bermudan swaptions in a LIBOR model. Journal of Derivatives, 11(3), 51-62.

Pietersz, R., Pelsser, A. A. J., \& Regenmortel, M. van (2004). Fast drift approximated pricing in the BGM model. Journal of Computational Finance, 8(1), 93-124.

Pietersz, R., \& Regenmortel, M. van (2006). Generic market models. Finance and Stochastics, 10(4), 507528.

Piterbarg, V. V. (2003). A practitioners guide to pricing and hedging callable LIBOR exotics in forward LIBOR models. SSRN Working Paper.

Piterbarg, V. V. (2004). Computing deltas of callable LIBOR exotics in forward LIBOR models. Journal of Computational Finance, 7(2), 107-144.

Piterbarg, V. V. (2004). TARNs: Models, valuation, risk sensitivities. Wilmott Magazine, 14, 62-71.

Rebonato, R. (1998). Interest rate option models (2nd ed.). Chichester: Wiley.

Rebonato, R. (1999). Volatility and correlation in the pricing of equity, FX and interest-rate options. Chichester: Wiley.

Rebonato, R. (2002). Modern pricing of interest-rate derivatives. New Jersey: Princeton University Press.

Ritchken, P., \& Sankarasubramanian, L. (1995). Volatility structures of the forward rates and the dynamics of the term structure. Mathematical Finance, 5(1), 55-72.

Rubinstein, M. (1983). Displaced diffusion option pricing. Journal of Finance, 38(1), 213-217.

Svenstrup, M. (2005). On the suboptimality of single-factor exercise strategies for Bermudan swaptions. Journal of Financial Economics, 78(3), 651-684.

Vasicek, O. (1977). An equilibrium characterization of the term structure. Journal of Financial Economics, 5(2), 177-188.

$\mathrm{Wu}$, L. (2003). Fast at-the-money calibration of the LIBOR market model using Lagrange multipliers. Journal of Computational Finance, 6(2), 39-77. 